\title{
Predicting persistent disease among children who wheeze during early life
}

\author{
R.J. Kurukulaaratchy*, S. Matthews*, S.T. Holgate*, S.H. Arshad*,\#
}

Predicting persistent disease among children who wheeze during early life. R.J. Kurukulaaratchy, S. Matthews, S.T. Holgate, S. H. Arshad. (C) ERS Journals Ltd 2003. ABSTRACT: This study sought to determine factors influencing the persistence of early life wheezing up to the age of 10 yrs and to create a score identifying those with the highest risk of persistent disease.

Children were seen at birth, 1, 2, 4 and $10 \mathrm{yrs}$ in a whole population birth cohort study $(n=1,456)$. Information was collected prospectively on wheeze prevalence and subjects were classified into wheezing phenotypes. Early life genetic and environmental risk factors were recorded and skin-prick testing (SPT) was performed at 4 yrs. Independently significant factors for persisting wheeze were identified at logistic regression and used to create a score for persistence.

Wheezing persistence from the first 4 yrs to the age of 10 yrs occurred in $37 \%$ of early life wheezers. Independent significance for persistence was associated with asthmatic family history, atopic SPT at 4 yrs and recurrent chest infections at 2 yrs, whilst recurrent nasal symptoms at 1 yr conferred reduced risk. A cumulative risk score using these factors identified wheezing persistence in $83 \%$ scoring 4 and transience in $80 \%$ scoring 0 .

Thus, a combination of genetic predisposition, early life atopy and recurrent chest infections favours the persistence of early life wheezing. Risk scores using such knowledge could provide prognostic guidance on the outcome of early wheeze.

Eur Respir J 2003; 22: 767-771.
*The David Hide Asthma \& Allergy Research Centre, St Mary's Hospital, Newport, Isle of Wight and ${ }^{\#}$ Respiratory Cell and Molecular Biology, School of Medicine, Southampton General Hospital, Southampton, UK.

Correspondence: S.H. Arshad, The David Hide Asthma \& Allergy Research Centre, St Mary's Hospital, Newport, Isle of Wight, PO30 5TG, UK.

Fax: 441983822928

E-mail: sha@soton.ac.uk

Keywords: Atopy, childhood asthma, persistent wheezing, risk factors, risk score

Received: January 162003

Accepted after revision: May 282003

This study was funded by the National Asthma Campaign, UK, grant number 364. The 10-yr follow-up was also funded with their assistance.
Over the past decade it has been increasingly recognised that childhood wheezing is a heterogeneous condition [1] rather than a single disease entity. While the common lay perception is that wheezing in early life follows a benign transient course, several cohort studies suggest otherwise. Thus, MARTINEZ et al. [2] demonstrated persistent wheezing up to the age of 6 yrs in $38 \%$ of early life wheezers; a similar proportion to that identified by BROOKE et al. [3] in their English cohort. The landmark Tucson study [2, 4] has shown that such persistent wheezers closely resemble the typical childhood asthmatic possessing high levels of personal atopy, impaired lung function and bronchial hyperresponsiveness (BHR). STEVENSON et al. [5] showed that ongoing "persistent inflammation" exists in the airways of typically asthmatic children even when asymptomatic. Persistent wheezers would therefore have the greatest scope for development of uninterrupted airway inflammation with possible long-term sequelae. Given the findings from the Childhood Asthma Management Program study [6] that showed a longer duration of wheezing to be associated with greater disease severity, such persistent wheezers therefore harbour significant potential for troublesome disease. Conversely, transient wheezers who constituted the remainder of early life wheezers in the Tucson study were found to show little atopy and BHR. In clinical practice, however, it is often difficult to accurately identify children who are likely to continue wheezing. Given the close parallels between most persistent wheezers and those diagnosed with childhood asthma, identification of factors in early life distinguishing persistent disease from a large body of coexistent

For editorial comments see page 719. transient wheeze is vital in improving childhood asthma management. Here, the findings from an unselected whole population birth cohort study, which provide a simple tool to help in the early identification of persistent childhood wheezing, are described.

\section{Methods}

A whole population birth cohort was established on the Isle of Wight in 1989 to prospectively study the natural history of childhood asthma and identify risk factors relevant to its development. Approval for the study was obtained from the Local Research Ethics Committee. Of 1,536 children born between 1 January 1989 and 28 February 1990, informed consent was obtained for 1,456 subjects to be enrolled. Enrolment took place at birth and information on family history of allergy (parental or sibling), household pets, smoking habit, social class (Registrar General's Classification) and birth weight were recorded. Cord blood immunoglobulin (Ig)E was measured in most instances at birth. Children were followed at the ages of $1 \mathrm{yr}(\mathrm{n}=1,167,80.2 \%), 2 \mathrm{yrs}(\mathrm{n}=1,174,80.6 \%)$, 4 yrs $(n=1,218,83.7 \%)$ and 10 yrs $(n=1,373,94.3 \%)$. The results of cohort follow-up (1, 2, 4 and 10 yrs) have been reported previously [7-10]. At every follow-up, detailed questionnaires were completed with the parents for each child regarding asthma and allergy prevalence. "Current wheeze" was recorded as having occurred in the prior 12 months. Exposure to relevant environmental factors (domestic pets and tobacco smoke) was noted. Method of feeding was obtained at 1 and 2 yrs. A history of recurrent chest 
infections (more than one in the past year) was determined on clinical grounds by the investigator at 1 and 2 yrs of age. Antibiotic usage was not a prerequisite for diagnosis of chest infection. Investigators' diagnoses of eczema (chronic or chronically relapsing, itchy dermatitis lasting $>6$ weeks with characteristic morphology and distribution), recurrent nasal symptoms/rhinitis (recurrent nasal discharge or blockage with attacks of sneezing and itchy eyes) and food allergy (history of vomiting, diarrhoea, colic or rash within $4 \mathrm{~h}$ of ingestion of a particular food on at least two occasions) were made each time. Skin-prick testing (SPT) was performed in most children seen at 4 yrs [9] $(n=981)$ to a panel of common inhaled and food allergens (Biodiagnostics, Reinbek, Germany). This comprised house dust mite (Dermatophagoides pteronyssinus), grass pollen mix, cat and dog epithelia, Alternaria alternata, Cladosporium herbarum, milk, hen's egg, soya, cod, wheat and peanut, plus histamine and physiological saline to act as positive and negative controls, respectively. Mean weal diameter of $\geqslant 3 \mathrm{~mm}$ greater than the negative control was regarded as a positive reaction.

\section{Analysis}

To improve accuracy, data double entry was performed with subsequent file comparison and reference to paper records to resolve any queries. Children were categorised at 10 yrs into four groups by presence of current wheeze at each follow-up: 1) nonwheezers (children who never wheezed during the first decade of life); 2) early transient wheezers (wheezing onset during the first 4 yrs of life that ceased and was not present within 12 months of assessment at $10 \mathrm{yrs}$ ); 3 ) persistent wheezers (children with wheezing onset during the first 4 yrs of life who still wheezed at $10 \mathrm{yrs}$ ); and 4) late-onset wheezers (children with wheezing onset from 5 yrs onwards who still wheezed at $10 \mathrm{yrs}$ ). To improve accuracy and minimise recall bias, the phenotypic analysis was restricted to $1,034(71 \%)$ of the original 1,456 cohort recruits who were seen prospectively with information available from all study visits, 1 or 2, 4 and 10 yrs. These 1,034 children did not differ significantly in a range of demographic parameters or relevant risk factors from 339 children seen at 10 yrs who were excluded from phenotypic analysis because of missing prior follow-up (table 1). Children included in the phenotypic analysis did experience significantly more breastfeeding $(\mathrm{p}=0.033)$ and less exposure to parental smoking at birth

Table 1.-Demographic/disease characteristics of children included and excluded from the analysis

\begin{tabular}{lcc}
\hline & Included $^{\#}$ & Excluded $^{\text {甲 }}$ \\
\hline Subjects n & 1034 & 339 \\
Male \% & 50.8 & 50.4 \\
Mean birth weight kg & 3.44 & 3.38 \\
Social class I-III at birth \% & 47.2 & 43.5 \\
Parental smoking at birth \% & 39.6 & $56.3^{* * *}$ \\
Cat ownership at birth \% & 34.1 & 30.2 \\
Dog ownership at birth \% & 29.7 & 26.3 \\
Exclusively breastfed & 35.2 & $27.6^{\S}$ \\
Parental smoking at 4 yrs \% & 37.2 & 40.5 \\
Atopic at 4-yr SPT \% & 19.8 & 19.4 \\
Parental asthma \% & 31.6 & 27.1 \\
Sibling asthma \% & 30.6 & 33.7 \\
Wheeze ever retrospective 10 yrs \% & 46.7 & 41.9 \\
Current wheeze at 10 yrs \% & 19.9 & 15.6 \\
\hline
\end{tabular}

${ }^{\#}$ : seen at all visits; ${ }^{\circ}$ : seen at 10 yrs but not at other visits; ${ }^{+}$: in first 3 months. SPT: skin-prick test. ${ }^{* * *}: \mathrm{p}<0.001$; ${ }^{\S}$ : $\mathrm{p}=0.033$. $(\mathrm{p}<0.001)$ than those excluded from analysis. This probably reflected the nonsignificant trend for higher social class in those included in the phenotypic analysis, since this factor was previously characterised as being associated with such findings in this cohort [7].

Univariate risk factor analysis was conducted to identify risk factors present in early life (the first $4 \mathrm{yrs}$ ) that were capable of predicting risk of wheezing persistence from early life up to $10 \mathrm{yrs}$ of age. Chi-squared analysis (with Fisher's exact test where indicated by low expected cell counts) was used for this purpose comparing factors between persistent and early transient wheezers. To obtain the independent effect of risk factors showing trends for significance at univariate testing $(\mathrm{p}<0.2)$, a logistic regression model was created. Stepwise backward (likelihood ratio) logistic regression was used for this purpose. Where more than one risk factor could explain a particular exposure of interest, only the most relevant was entered into the model. Factors demonstrating independent significance for wheezing persistence were then selected to compile a cumulative risk score that could provide a stratified assessment of persistent wheezing risk. Thus, if no factors were present the subject's risk score was zero, if one was present the score was one, two present the score was two etc. Sensitivity, specificity, positive predictive values, negative predictive values and likelihood ratios at successive strata of the risk score were calculated.

\section{Results}

By $10 \mathrm{yrs}$, the cumulative prevalence of prospectively determined "wheeze ever" was 40.3\% (417 children). Of these, 336 started to wheeze before the age of 4 yrs. Among such early childhood wheezers, $211(63 \%)$ were early transient wheezers and $125(37 \%)$ persistent wheezers.

Factors showing trends for significance $(\mathrm{p}<0.2)$ at univariate analysis for persistence of early life wheezing are shown in table 2. Personal histories of eczema at $1 \mathrm{yr}$, recurrent chest infections at 2 yrs plus eczema, and rhinitis at 4 yrs all conferred significant risk of wheezing persistence. Atopic sensitisation at 4 yrs also significantly increased the risk of persistent wheezing. A nonsignificant trend in favour of transient wheezing was found with recurrent nasal symptoms at $1 \mathrm{yr}$. Family history of asthma (defined as present in parent or sibling) carried a significantly increased risk of wheezing persistence while paternal eczema gave a nonsignificant effect in the same direction. Wheezing persistence was significantly associated with maternal smoking at birth and parental smoking at 4 yrs at univariate testing. Neither sex nor social class at birth had significant influence upon wheezing persistence whilst cord IgE did not differ significantly between persistent and early transient wheezers (median 0.1 versus 0.1 , respectively; $\mathrm{p}=0.298$ Mann Whitney U-test).

In the final logistic regression model used, multivariate analysis (table 3) identified independent significance for wheezing persistence with a family history of asthma, recurrent chest infections at 2 yrs and atopic SPT at 4 yrs. A similar but nonsignificant trend for wheezing persistence was observed with formula feeding $(\mathrm{p}=0.094$, odds ratio (OR) $1.67,95 \%$ confidence interval (CI) 0.92-3.06). Conversely, a significantly reduced risk of wheezing persistence was found with recurrent nasal symptoms at 1 yr. Similar nonsignificant trends in favour of transient wheezing were found for cat ownership at birth $(\mathrm{p}=0.091$, OR $0.58,95 \%$ CI $0.30-1.09)$ and sibling eczema ( $\mathrm{p}=0.074$, OR $0.56,95 \%$ CI $0.29-1.06$ ).

The four factors demonstrating independent significance for increased wheezing persistence had varying prevalence 
Table 2. - Univariate risk for persistence of early life wheezing

\begin{tabular}{|c|c|c|c|c|c|}
\hline \multirow[t]{2}{*}{ Factor } & \multicolumn{2}{|c|}{ Prevalence in phenotype } & \multirow[t]{2}{*}{ p-value } & \multirow[t]{2}{*}{ OR } & \multirow[t]{2}{*}{$95 \% \mathrm{CI}$} \\
\hline & Persistent & Transient & & & \\
\hline Atopic SPT at 4 yrs & $46.8(52 / 111)$ & $12.6(23 / 182)$ & $<0.001^{*}$ & 6.09 & $3.43-10.82$ \\
\hline Eczema at $1 \mathrm{yr}$ & $27.2(34 / 125)$ & $14.2(30 / 211)$ & $0.003^{*}$ & 2.25 & $1.30-3.91$ \\
\hline Nasal symptoms at $1 \mathrm{yr}$ & $14.4(18 / 125)$ & $22.3(47 / 211)$ & 0.077 & 0.59 & $0.32-1.07$ \\
\hline Food allergy at $1 \mathrm{yr}$ & $16.8(21 / 125)$ & $10.4(22 / 211)$ & 0.091 & 1.74 & $0.91-3.30$ \\
\hline Food allergy at 2 yrs & $8.8(10 / 114)$ & $4.5(9 / 199)$ & 0.130 & 2.03 & $0.80-5.15$ \\
\hline Eczema at 4 yrs & $23.4(29 / 124)$ & $8.1(17 / 210)$ & $<0.001^{*}$ & 3.47 & $1.81-6.62$ \\
\hline Rhinitis at 4 yrs & $16.8(21 / 125)$ & $6.2(13 / 210)$ & $0.002 *$ & 3.06 & $1.47-6.36$ \\
\hline Family history asthma & $71.3(87 / 122)$ & $52.6(101 / 192)$ & $0.001^{*}$ & 2.24 & $1.38-3.63$ \\
\hline Parental asthma & $45.9(55 / 122)$ & $34.9(67 / 192)$ & 0.051 & 1.58 & $1.00-2.52$ \\
\hline Sibling asthma & $42.6(52 / 122)$ & $34.2(66 / 193)$ & 0.132 & 1.43 & $0.90-2.28$ \\
\hline Paternal eczema & $12.4(15 / 121)$ & $6.8(13 / 192)$ & 0.089 & 1.95 & $0.89-4.25$ \\
\hline Sibling eczema & $31.1(38 / 122)$ & $38.9(75 / 193)$ & 0.164 & 0.71 & $0.44-1.15$ \\
\hline Exclusively breastfed $^{\#}$ & $20.0(24 / 120)$ & $28.6(55 / 192)$ & 0.088 & 0.62 & $0.36-1.08$ \\
\hline Exclusively formula fed $^{\#}$ & $49.2(59 / 120)$ & $39.1(79 / 202)$ & 0.078 & 1.51 & $0.95-2.38$ \\
\hline Maternal smoking at birth & $33.0(41 / 123)$ & $23.0(48 / 209)$ & $0.039^{*}$ & 1.68 & $1.02-2.75$ \\
\hline Parental smoking at birth & $52.0(64 / 123)$ & $44.0(91 / 207)$ & 0.155 & 1.38 & $0.88-2.16$ \\
\hline Parental smoking at $1 \mathrm{yr}$ & $51.2(64 / 125)$ & $42.1(88 / 209)$ & 0.106 & 1.44 & $0.92-2.25$ \\
\hline Parental smoking at 4 yrs & $52.8(66 / 125)$ & $40.5(85 / 210)$ & $0.028 *$ & 1.65 & $1.05-2.57$ \\
\hline Chest infections at 2 yrs & $42.1(48 / 114)$ & $29.1(58 / 199)$ & $0.020 *$ & 1.77 & $1.09-2.86$ \\
\hline Cat owned at birth & $26.6(33 / 124)$ & $33.8(71 / 210)$ & 0.170 & 0.71 & $0.44-1.16$ \\
\hline Dog owned at birth & $24.2(30 / 124)$ & $31.4(66 / 210)$ & 0.158 & 0.70 & $0.42-1.15$ \\
\hline
\end{tabular}

Data are presented as \% (n/total n). \#: in first 3 months; SPT: skin-prick test; OR: odds ratio; CI: confidence interval. Only factors showing trends for significance at univariate analysis $(\mathrm{p}<0.2)$ are shown. Factors tested but not showing trends for significance were nasal symptoms at 2 yrs, eczema at $2 \mathrm{yrs}$, food allergy at $4 \mathrm{yrs}$, individual maternal/paternal asthma, maternal eczema, family history of rhinitis (maternal/paternal/sibling/combined), family history of food allergy (maternal/paternal/sibling/combined), family history of urticaria (maternal/paternal/sibling/combined), early weaning (first 3 months), recurrent chest infections at $1 \mathrm{yr}$, cat ownership at 1,2 and 4 yrs, and dog ownership at 1,2 and 4 yrs. *: p $<0.05$ persistent versus transient outcome.

Table 3. - Multivariate analysis for the persistence of early life wheezing

\begin{tabular}{lrrl}
\hline Risk factors & p-value & OR & 95\% CI \\
\hline Recurrent chest infections at 2 yrs & 0.034 & 1.99 & $1.05-3.77$ \\
Family history of asthma & 0.010 & 2.31 & $1.22-4.37$ \\
Atopic SPT at 4 yrs & $<0.001$ & 5.73 & $2.95-11.12$ \\
Nasal symptoms at 1 yr & 0.039 & 0.43 & $0.19-0.96$ \\
\hline
\end{tabular}

Risk factors for persistence of early life wheezing refer to comparison of risk factors for persistent with those for early transient wheezing. OR: odds ratio; CI: confidence interval. Only factors that showed a significant effect at the final step of the regression model are shown. Factors entered into the regression model were atopic skin-prick test (SPT) at 4 yrs, recurrent chest infections at 2 yrs, exclusively formula fed, food allergy at $1 \mathrm{yr}$, food allergy at $2 \mathrm{yrs}$, nasal symptoms at $1 \mathrm{yr}$, cat owned at birth, dog owned at birth, family history of asthma, sibling eczema, eczema at $1 \mathrm{yr}$, eczema at $4 \mathrm{yrs}$, rhinitis at $4 \mathrm{yrs}$, maternal smoking at birth, parental smoking at $1 \mathrm{yr}$ and parental smoking at $4 \mathrm{yrs}$. among early life wheezers. Thus, asthmatic family history occurred in $59.9 \%$, recurrent chest infections at 2 yrs in $33.9 \%$, atopic SPT at $4 \mathrm{yrs}$ in $25.6 \%$ and absence of nasal symptoms at $1 \mathrm{yr}$ in $80.7 \%$. These factors were used to create a cumulative risk score for symptom persistence. Among early life wheezers $3.9 \%$ had a score of $0,26.7 \%$ a score of $1,39.6 \%$ a score of $2,25.1 \%$ a score of 3 and $4.7 \%$ a score of 4 . The prevalence and risk of wheezing persistence for this scoring system is shown in table 4 . Risk scores of 3 and 4 were both associated with significantly increased risk of symptom persistence with $83 \%$ of those scoring 4 having persistent disease. Scores of 0,1 or 2 were associated with either a trend for or statistical significance for transient disease. Among children with a zero risk score, $80 \%$ had a transient wheezing outcome. Sensitivity, specificity, positive predictive values, negative predictive values and likelihood ratios for successive strata of the risk score are given in table 5 . A risk score of 3 or 4 was three times as likely to occur in a wheezing child destined to suffer persistent disease than one who would outgrow their condition.

Table 4.-Prevalence and risk of wheezing outcome according to risk scoring system

\begin{tabular}{|c|c|c|c|c|c|}
\hline \multirow[t]{2}{*}{ Risk score } & \multicolumn{2}{|c|}{ Wheezing outcome for each score } & \multirow[t]{2}{*}{ p-value } & \multirow[t]{2}{*}{ OR } & \multirow[t]{2}{*}{$95 \% \mathrm{CI}$} \\
\hline & Persistent & Transient & & & \\
\hline 0 & $20.0(2 / 10)$ & $80.0(8 / 10)$ & $0.324^{\#}$ & 0.38 & $0.08-1.83$ \\
\hline 1 & $19.1(13 / 68)$ & $80.9(55 / 68)$ & $<0.001$ & 0.28 & $0.14-0.54$ \\
\hline 2 & $31.7(32 / 101)$ & $68.3(69 / 101)$ & 0.058 & 0.60 & $0.36-1.02$ \\
\hline 3 & $65.6(42 / 64)$ & $34.4(22 / 64)$ & $<0.001$ & 4.49 & $2.46-8.19$ \\
\hline 4 & $83.3(10 / 12)$ & $16.7(2 / 12)$ & $0.002^{\#}$ & 8.65 & $1.85-40.38$ \\
\hline
\end{tabular}

Data are presented as \% (n/total $\mathrm{n})$. OR: odds ratio; CI: confidence interval. Values shown indicate wheezing outcome at varying degrees of risk score. $\#$ : comparisons are of persistent and transient wheezing outcome by Chi-squared analysis with Fisher's exact test where indicated by low expected cell counts. 
Table 5. - Sensitivity, specificity, predictive values and likelihood ratios for risk scoring system

\begin{tabular}{lccccc}
\hline Risk score strata & Sensitivity & Specificity & Positive predictive value & Negative predictive value & Likelihood ratio $^{\#}$ \\
\hline$\geqslant 1$ & 0.979 & 0.051 & 0.396 & 0.800 & 1.032 \\
$\geqslant 2$ & 0.845 & 0.404 & 0.475 & 0.808 & 1.418 \\
$\geqslant 3$ & 0.525 & 0.846 & 0.684 & 0.737 & 3.409 \\
$=4$ & 0.103 & 0.987 & 0.833 & 0.639 & 7.923 \\
\hline
\end{tabular}

Results for each risk score level refer to findings where the score concerned is regarded as a positive test result and scores below that are regarded as negative results. ${ }^{\#}$ : likelihood ratio refers to sensitivity/(1-specificity).

\section{Discussion}

Among the prospectively followed cohort, $33 \%$ of the population had wheezed in the first 4 yrs of life. While most of these children were seen to lose their symptoms by the age of $10 \mathrm{yrs}$, a substantial minority $(37 \%)$ developed a persistent form of disease that may be considered typical of childhood asthma. A combination of an asthmatic family history, recurrent chest infections in the second year of life, atopic sensitisation at 4 yrs of age and absence of recurrent nasal symptoms in the first year of life were identified to confer high risk of disease persistence in a child who wheezes during early life.

In this context genetic predisposition towards being atopic and asthmatic would appear to be a crucial feature of wheezing persistence, confirming results such as those of KOEPPEN-SCHOMERUS et al. [11] who, in a recent study of twin pairs, showed that heritable factors predominate over shared environmental influences in asthma development. The present findings add to those of CsONKA et al. [12] who retrospectively looked at risk factors for wheezing persistence in their study group, and confirm the importance of heredity towards the persistent asthmatic phenotype suggested by LONDON et al. [13]. These results are also consistent with findings emerging from the Multicentre Asthma Study (MAS) [14] that identified an interaction of persistent atopic sensitisation in childhood and parental atopy/asthma as being associated with childhood asthma development. However, it is also worth considering whether some degree of such familial disease clustering may arise not only from genetic influences, but also partly from shared environment in early life.

One drawback of a study like this is of not being able to provide an unequivocal answer in this regard. Certainly it is unlikely that heredity alone can explain the development of wheezing persistence. The present findings suggest that in addition to genetic predisposition exposures like recurrent chest infections in infancy also significantly influence wheezing persistence. STEIN et al. [15] demonstrated an association between respiratory syncytial virus infection in early life with both frequent and infrequent early life wheezing illnesses. However, the influence of such early life infection was seen to wane with age in that study. In this study, a continued influence for early life chest infection was identified for wheezing persistence as far as $10 \mathrm{yrs}$. This finding may be consistent with past speculation over the consequences [15, 16] of such infections upon bronchial epithelium and may be one reason for wheezing persistence at a structural level.

An alternative interpretation may be that asthmatic childhood phenotypes more readily manifest lower respiratory tract symptoms when infected. Therefore, rather than causing persistent wheeze, such infections may simply be a reflection of its presence. Recurrent nasal symptoms in the first year of life (probably reflecting upper respiratory tract viral infections) were found to favour a transient wheezing outcome. This would be consistent with findings from the MAS [17] that 1 -yr-olds with recurrent nasal symptoms were less likely to suffer subsequent asthma and atopy. These data did not demonstrate a significant independent effect of other environmental exposures such as parental smoking, social class, pet exposure or method of infant feeding upon persistence of early life wheezing. However, nonsignificant trends were seen for increased wheezing persistence with exclusive formula feeding and a transient wheezing outcome with cat ownership at birth.

Previously, CASTRO-Rodriguez et al. [18] created a clinical index to identify asthma risk among recurrently wheezing young children in the Tucson cohort study. Using that system, cases identified with a positive stringent index carried a prevalence of $76 \%$ for active asthma while $95 \%$ of those with a negative stringent index never had active asthma. Here, the authors attempted to create a clinical risk score that could be used to predict the risk of symptom persistence in early wheezers using risk factors that were demonstrated to have independent significance for this outcome. Thus, attention was focused upon what were felt to be the most relevant genetic and environmental factors that may influence wheezing persistence in the population. Such an approach may be usefully augmented by addition of data concerning severity and morbidity of wheezing in early life. Unfortunately such data was not prospectively available in early life to be included in the prospectively determined risk score.

The information needed to calculate the score is easily obtainable by a clinician in an outpatient setting. The emphasis is on an accurate history in combination with SPT at 4 yrs. In this context it is worth considering whether atopic status in earlier childhood rather than at 4 yrs may be more informative in differentiating wheezing outcome. Insufficient SPT in infancy was available in this study to allow meaningful analysis of significance and thus inclusion in the scoring system but this point is worth considering in future work. Some may advocate a clinical definition of atopy rather than including SPT in such a scoring system. However, it should be remembered that SPT is safe, reproducible and relatively simple to perform even in a small child. It is a test that certainly could be incorporated into most paediatric outpatient settings and may even find usage in a general practitioner-run asthma clinic. Importantly, its presence in the score permits an objective measure to be included.

With this risk score, a maximum score of 4 was associated with prevalence for wheezing persistence of $83 \%$ while a zero score was associated with prevalence for transient wheezing of $80 \%$. However, such scores at either extreme were present in only a few subjects. Rather than attempting to devise a composite dichotomous system of high or low score (i.e. negative or positive test), the authors have simply described the risk at successive strata of the score. The advantage of this is that the parent and physician can easily estimate the risk of persistence for a child with early onset wheeze. For instance, a score of 3 or more was found to be three times as likely in a child with wheezing persistence than a transient disease outcome. Such a scoring system may provide a simple but useful guide in the clinical setting to improve early identification of those children most at risk of wheezing persistence. Thus clinicians 
may consider a lower threshold for use of regular prophylactic therapy such as inhaled corticosteroids in children with early life wheezing possessing persistence scores of 3 or 4 by the age of 4 yrs. This score may also help prevent inappropriate use of unnecessary medication in wheezing children who actually have a low risk of disease persistence. Clinicians should find it helpful to be able to quantify the degree of persistence risk as outlined by this score when discussing issues like commencement of regular therapy with the parent. This in turn may lead to better compliance with regular treatment when it is really needed.

Finally, it is worth reflecting that the tools in this risk score permit its effective use at 4 yrs of age. However, even earlier identification of wheezing persistence may ultimately prove more useful, since this may provide even further clarity and perhaps permit earlier intervention. The need to focus on very early life in this context should therefore be a matter of priority in future work of this kind.

\begin{abstract}
Acknowledgements. The authors gratefully acknowledge the cooperation of the children and parents who have participated in this ongoing study. They also note the valued contributions of L. Terry, G. Poulton, M. Fenn, L. Waterhouse, H. Savory, T. Booth, A. Gallini, C. Wilby, R. Lisseter and R. Twiselton to the 10-yr follow-up of this study. Finally, they would like to highlight the role of the late D. Hide in conceiving this study.
\end{abstract}

\section{References}

1. Martinez FD, Helms PJ. Types of asthma and wheezing. Eur Respir J 1998; 12: Suppl. 27, 3s-8s.

2. Martinez FD, Wright AL, Taussig LM, Holberg CJ, Halonen M, Morgan WJ. Asthma and wheezing in the first six years of life. $N$ Engl $J$ Med 1995; 332: 133-138

3. Brooke AM, Lambert PC, Burton PR, Clarke C, Luyt DK, Simpson H. The natural history of respiratory symptoms in preschool children. Am J Respir Crit Care Med 1995; 152: $1872-1878$.

4. Stein RT, Holberg CJ, Morgan WJ, et al. Peak flow variability, methacholine responsiveness and atopy as markers for detecting different wheezing phenotypes in childhood. Thorax 1997; 52: 946-952.

5. Stevenson EC, Turner G, Heaney LG, et al. Bronchoalveolar lavage findings suggest two different forms of childhood asthma. Clin Exp Allergy 1997; 27: 1027-1035.

6. Zeiger RS, Dawson C, Weiss S. Relationships between duration of asthma and asthma severity among children in the Childhood Asthma Management Program (CAMP). J Allergy Clin Immunol 1999; 103: 376-387.

7. Arshad SH, Hide DW. Effect of environmental factors on the development of allergic disorders in infancy. J Allergy Clin Immunol 1992; 90: 235-241.

8. Arshad SH, Stevens M, Hide DW. The effect of genetic and environmental factors on the prevalence of allergic disorders at the age of two years. Clin Exp Allergy 1993; 23: 504-511.

9. Tariq SM, Matthews SM, Hakim EA, Stevens M, Arshad SH, Hide DW. The prevalence of and risk factors for atopy in early childhood: a whole population birth cohort study. J Allergy Clin Immunol 1998; 101: 587-593.

10. Kurukulaaratchy RJ, Fenn M, Twiselton R, Matthews S, Arshad SH. The prevalence of asthma and wheezing illnesses amongst 10-year old schoolchildren. Respir Med 2002; 96: $162-169$.

11. Koeppen-Schomerus G, Stevenson J, Plomin R. Genes and environment in asthma: a study of 4 year old twins. Arch Dis Child 2001; 85: 398-400.

12. Csonka P, Kaila M, Laippala P, Kuusela A-L, Ashorn P Wheezing in early life and asthma at school age: Predictors of symptom persistence. Pediatr Allergy Immunol 2000; 11: $225-229$

13. London SJ, James Gauderman W, Avol E, Rappaport EB, Peks JM. Family history and the risk of early-onset persistent, early-onset transient and late onset asthma. Epidemiology 2001; 12: 577-583.

14. Illi S, von Mutius E, Lau S, et al. The pattern of atopic sensitisation is associated with the development of asthma in childhood. J Allergy Clin Immunol 2001; 108: 709-714.

15. Stein RT, Sherill D, Morgan WJ, et al. Respiratory syncytial virus in early life and risk of wheeze and allergy by age 13 years. Lancet 1999; 354: 541-545.

16. Douglass JA, $\mathrm{O}^{\prime}$ Hehir RE. What determines asthma phenotype? Respiratory infections and asthma. Am J Respir Crit Care Med 2000; 161: s211-s214.

17. Illi S, von Mutius E, Lau S, et al. Early childhood infectious diseases and the development of asthma up to school age: a birth cohort study. BMJ 2001; 322: 390-395.

18. Castro-Rodriguez JA, Holberg CJ, Wright AL, Martinez FD. A clinical index to define risk of asthma in young children with recurrent wheezing. Am J Respir Crit Care Med 2000; 162: 1403-1406. 